\section{Ein Zellkulturfermenter mit integriertem Membransystem für die homogene Kultur- führung*}

\author{
Heino Büntemeyer, Berthold G. D. Bödeker \\ und Jürgen Lehmann**
}

Der zur Zeit herrschende Trend zur in vitro-Kultur tierischer bzw. humaner Zellen erfordert durch die Fortschritte der Gentechnologie leistungsfähige Zellkultur-Reaktoren. Bisher wurden eine Vielzah unterschiedlichster Reaktortypen entwickelt, die im wesentlichen in die zwei Gruppen homogener bzw. inhomogener Zellverteilung eingeordnet werden können.

Das in dieser Arbeit beschriebene Fermentationssystem verbindet die jeweiligen Vorzüge der homogenen (z. B. Rührkessel) und der inhomogenen (Dichtzellreaktor) Zellverteilung. Die Vorteile eines homogenen Fermenters liegen in der einfachen Kontrolle der Zellen und der Kulturbedingungen ( $\mathrm{pO}_{2}$-Wert, $\mathrm{pH}$-Wert, Substratkonzentration usw.), während beim Dichtzellreaktorsystem hohe Zellkonzentrationen in der Regel für hohe Produktkonzentrationen sorgen.

\section{Fermenteraufbau und Funktionsprinzip}

Das vorgestellte Konzept besteht aus einem Rührreaktor mit einem Membransystem, das sowohl im, wie auch außerhalb des Fermenters angebracht ist (Abb. 1). Innerhalb des Reaktors sind zwei getrennte Membransysteme in Form von Hohlfäden auf dem Rührer angebracht, die zum einen für die Begasung sorgen und zum anderen eine Mikrofiltration der Kultursuspension erlauben (Doppelmembranrührer) [1-3].

Je nach den Sauerstoff-Bedürfnissen der verwendeten Zellinie werden für die Begasung 1 bis $2 \mathrm{~m}$ hydrophobe Membranhohlfäden aus mikroporösem Polypropylen (Tab. 1) (Accurel, ENKA, Wuppertal) pro Liter Fermentationsbrühe in den Fermenter eingebracht. An den Eingang (Abb. 2) ist eine Gasversorgungseinheit angeschlossen, die in Abhängigkeit von $\mathrm{pH}$ - und $\mathrm{pO}_{2}$-Wert der Kultur ein Gasgemisch aus Luft, $\mathrm{N}_{2}$, $\mathrm{O}_{2}$ und $\mathrm{CO}_{2}$ erzeugt, das jederzeit zur optimalen Versorgung der Kultur geeignet ist. Gleichermaßen kann von der Kultur, besonders bei hoher Zelldichte, gebildetes $\mathrm{CO}_{2}$ durch die Membran ausgetragen werden. Um das Druckgleichgewicht an der flüssigseitigen Membranoberfläche (Flüssigkeitsdruck, Kopfdruck, Membraneingangs- und -ausgangsdruck) so zu stabilisieren, daß keine Kultursuspension in die Poren eindringen kann, ist ein Uberströmregler am

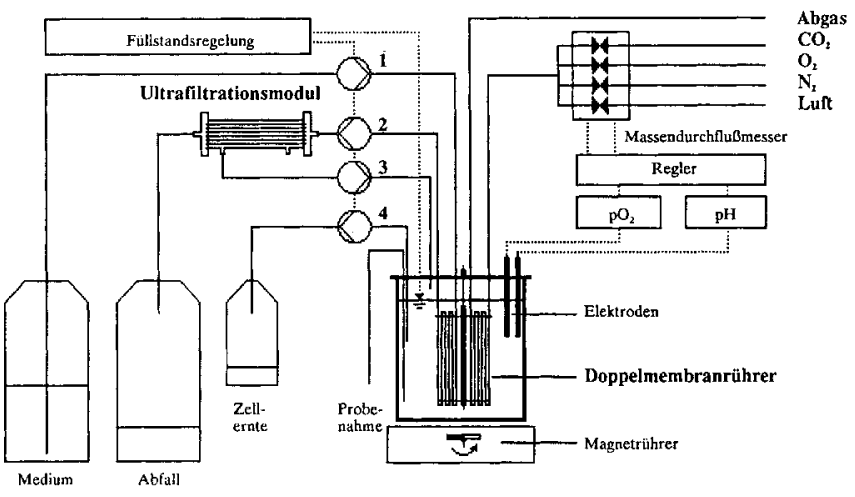

Abb. 1. Schematischer Gesamtaufbau des Fermentationssystems; 1 Medienpumpe, 2 Erntepumpe, 3 Rückführungspumpe, 4 Zellerntepumpe.

* Vortrag von $H$. Büntemeyer auf dem Jahrestreffen der VerfahrensIngenieure, 27. bis 29. Sept. 1989 in Berlin.

** Dr. H. Büntemeyer und Prof. Dr.-Ing. J. Lehmann, Zellkulturtechnik, Technische Fakultät der Univ. Bielefeld, Postfach 86 40, 4800 Bielefeld 1, Dr. B. G. D. Bödeker, Bayer AG, 5600 WuppertalElberfeld.
Tabelle 1.

Daten der Accurel-Hohlfaser-Membran (ENKA, Wuppertal).

$\begin{array}{llc}d_{\mathrm{a}}=2,6 \mathrm{~mm} d_{\mathrm{i}}=1,8 \mathrm{~mm} & & \\ \text { Porosität } & : & 75,0 \% \\ \text { mittlere Porengröße } & : & 0,3 \mu \mathrm{m} \\ \text { Berstdruck } & : & 6,5 \mathrm{bar} \\ \text { Implosionsdruck } & : & 1,5 \mathrm{bar} \\ \text { spez. Oberfläche, außen } & : & 81,7 \mathrm{~cm}^{2} / \mathrm{m} \\ \text { spez. Oberfläche, innen } & : & 56,5 \mathrm{~cm}^{2} / \mathrm{m} \\ \text { spez. Volumen, außen } & : & 5,3 \mathrm{ml} / \mathrm{m} \\ \text { spez. Volumen, innen } & : & 2,5 \mathrm{ml} / \mathrm{m}\end{array}$

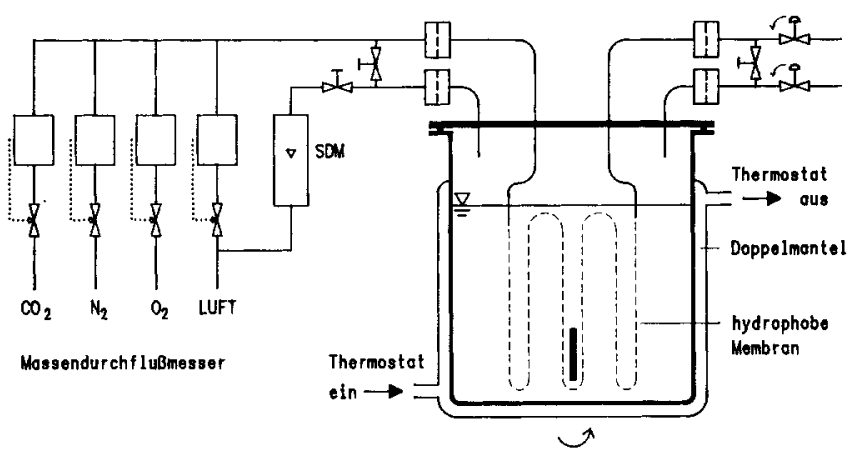

Abb. 2. Aufbau und Funktionsweise des Begasungssystems.

Membranausla $B$ angebracht und auf geringen Überdruck innerhalb der Membran eingestellt.

Das zweite Membransystem im Fermenter besteht ebenfalls aus mikroporösen Polypropylen-Hohlfäden (s. o.), die allerdings zuvor mit Ethanol hydrophilisiert wurden. Um eine Langzeitfiltration zu gewährleisten, wird diese Membran nach einem speziellen Modus betrieben (Abb. 3). Dazu sind an Membraneingang und -ausgang peristaltische Pumpen angeschlossen, die immer nur wechselseitig betrieben werden. Während die Erntepumpe steht und den Ausgang der Membran verschließt, fördert die Medienpumpe frisches Kulturmedium durch die Membran in den Fermenter. Beim Ernten verschließt die Medienpumpe den Membraneingang, so daß zellfreie Kultursuspension durch die Membranporen $(0,3 \mu \mathrm{m})$ aus dem Fermenter

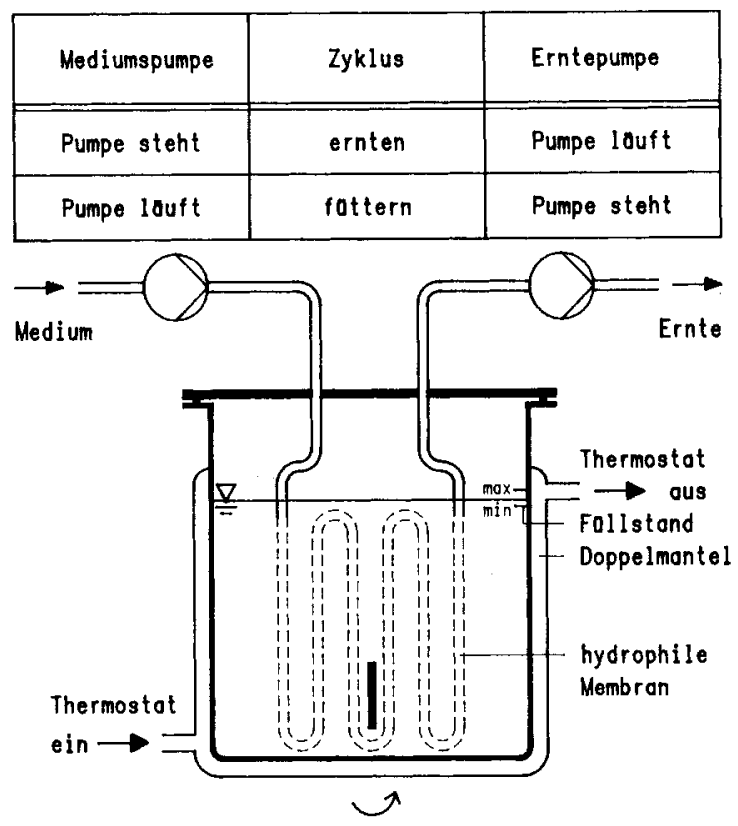

Abb. 3. Aufbau und Funktionsweise des Mikrofiltrationssystems. 
gepumpt werden kann. Ein kompletter Zyklus aus Auffüllen/ Rückspülen und Ernten/Filtrieren dauert je nach Fermentervolumen 20 bis 30 min, wobei das Auffülen/Rückspülen davon nur 10 bis $20 \%$ der Zeit in Anspruch nimmt.

Die Langzeitstabilität wird durch folgende Maßnahmen aufrecht erhalten: 1) hoher flächenbezogener Volumenstrom beim Rückspülen mit frischem Medium, 2) niedriger flächenbezogener Volumenstrom beim Filtrieren, 3) maximaler Durchsatz von 1 I pro m Membran und Tag.

Der mikrofiltrierte, zellfreie Uberstand wird direkt in eine außerhalb des Fermenters angebrachte Ultrafiltrationseinheit (Abb. 1) gegeben. Während das Filtrat dieser Einheit verworfen wird, kann das Retentat zur Aufarbeitung oder zurück in den Fermenter geführt werden. Je nach AusschluBgrenze der UF-Membran enthält das Retentat neben dem gewünschten Produkt alle Proteine des eingesetzten, vorzugsweise serumfreien Mediums. Ist das Produkt stabil und hemmt seine eigene Produktion nicht, bringt die Rückführung des gesamten Retentats den Vorteil, daß im Laufe der Fermentation sich das Verhältnis von Zielprotein (Produkt) zu Fremdprotein (Mediumbestandteile) immer mehr verbessert und die spätere Aufarbeitung erleichtert wird. Durch die gleichzeitige Rückführung der eingesetzten Proteine des serumfreien Mediums können die Medienkosten nahezu auf die Kosten des reinen Basalmediums reduziert werden [4].

Mit einer weiteren Pumpe kann im Bedarfsfall komplette Kultursuspension aus dem Fermenter entnommen werden. Dies wird erforderlich, wenn sich zu viele tote Zellen ansammeln oder aber auch, wenn lebende Zellen geerntet werden sollen.

\section{Material und Methoden}

Das beschriebene Fermentatiossystem wurde mit der EBV-transformierten humanen B-Zellinie $\mathrm{E}_{4}$ (ATCC-Nr.: CRL 8562) getestet, die einen humanen Antikörper IgM gegen Pseudomonas aeruginosa produziert. Die Zellen wurden in einem serumfreien Medium, bestehend aus einer 1:1-Mischung von Dulbecco's modifiziertem Eagle-Medium DMEM und Ham's F12 (Gibco, Eggenstein) unter Zusatz einer Anzahl hochmolekularer Komponenten (Rinderserumalbumin, Insulin, Transferrin) und diverser niedermolekularer Substanzen (Gluta$\min (+2 \mathrm{mmol} / 1)$, Olsäure, Cholesterin, Etanolamin, Biotin, Glutathion, Vitamin E-Acetat, Mercaptoethanol, Selendioxid) gezüchtet. Glucose und Lactat wurden mit halbautomatischen Analyzern (YSI Model 27A, Yellow Springs Instruments, Ohio/USA) bestimmt. Die Aminosäuren wurden über Reversed phase-HPLC nach Derivatisierung mit o-Phthaldialdehyd quantifiziert. Die Antikörper-Konzentration wurde über ELISA-Technik ermittelt [5].

\section{Ergebnisse}

Abb. 4 zeigt das Ergebnis der Kultivierung der $\mathrm{E}_{4}$-Zelle. In einem 1,5-1Fermenter mit einem Arbeitsvolumen von 1,2 I wurden je $2 \mathrm{~m}$ hydrophilisierte und hydrophobe Membran auf dem Doppelmembranrührer aufgebracht. Das Konzentrat aus dem UF-Modul (Cutoff: 10000 Dalton) wurde zurück in den Fermenter geführt.

Die Zellen wurden zu einer Anfangslebendzelldichte von 5,5·10 $\mathrm{Zel}$ len/ml inokuliert. Nach einem Tag Batch-Phase wurde der Perfusionsbetrieb aufgenommen. Mit dem Wachstum der Zellen im Laufe der Fermentation wurde allmählich die Perfusionsrate angehoben, aber nicht über $1,2 \mathrm{~d}^{-1}$ gesteigert, um ein Verblocken der Membran zu verhindern.

An den Tagen 9, 12 und 14 wurden batchweise $50 \%$ des Fermenterinhaltes komplett entfernt und durch frisches Komplettmedium, allerdings ohne Proteinzusatz, ersetzt. Ab Tag 18 wurde kontinuierlich Zellsuspension entfernt.

Da sämtliche Proteine (Insulin nur zum Teil) durch das UF-Modul zurückgeführt wurden, war das Perfusionsmedium völlig proteinfrei und enthielt als Zusätze nur die niedermolekularen Supplemente. Während die spezifische Wachstumsrate $\mu$ ab Tag 2 einen annähernd
Fermentation des hu.EBV-tr. B-Lymphozyten E4 im 1.2 I - Rührreaktor

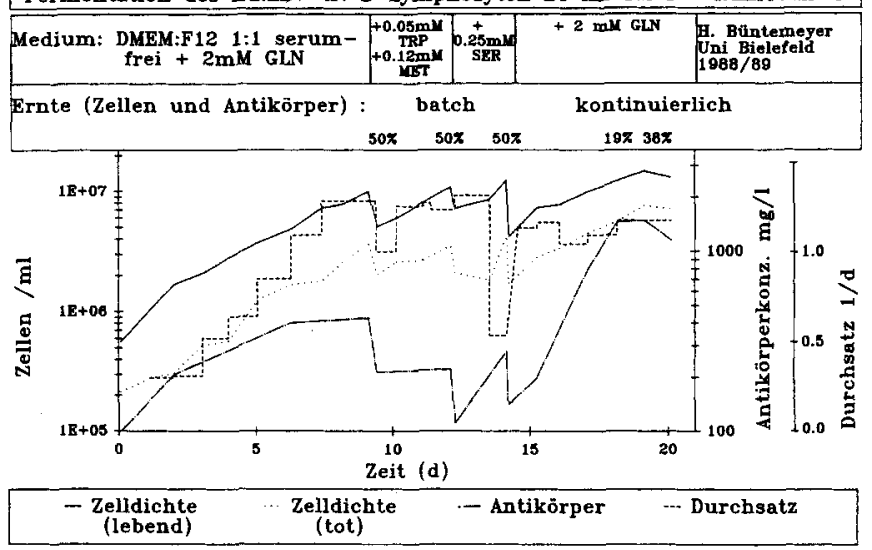

Abb. 4. Fermentation der Zellinie $\mathrm{E}_{4}$ im 1,2-1-Perfusionsfermenter.

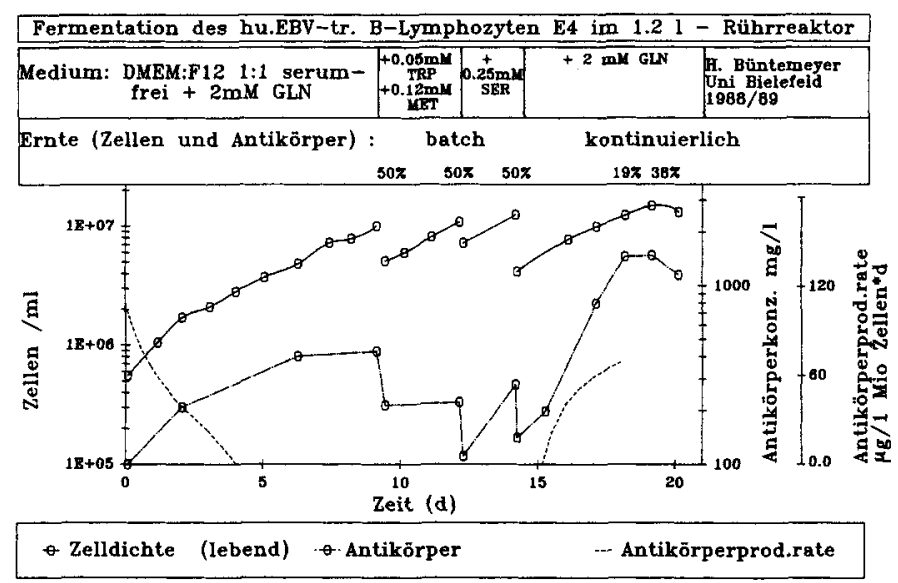

Abb. 5. Änderung der spezifischen Raten im Laufe der Fermentation.

konstanten Wert von $\mu=0,01 \mathrm{~d}^{-1}$ annimmt (Abb. 5), sinkt im ersten Abschnitt der Fermentation (bis Tag 9) die spezifische IgMProduktionsrate $q_{\mathrm{IgM}}$ auf Null ab. Durch die Rückführung ergibt sich eine IgM-Konzentration von etwa $400 \mathrm{mg} / \mathrm{l}$. Die Einstellung der Antikörper-Produktion wird erst verständlich, wenn eine umfangreiche Analyse der Substrate durchgeführt wird. In diesem Fall zeigt sich ab Tag 3 eine konstante Konzentration der Aminosäuren Tryptophan, Methionin und Serin. Obwohl die Konzentrationswerte nicht auf Null absinken, liegt die Vermutung nahe, daß eine Limitierung einer oder mehrerer dieser Aminosäuren vorliegt. Aus diesem Grunde wurde das Perfusionsmedium ab Tag 9 zusätzlich mit $90 \mu \mathrm{mol} / 1$ Tryptophan und $240 \mu \mathrm{mol} / \mathrm{l}$ Methionin supplementiert. Dieser Zusatz hat allerdings keinerlei Auswirkung auf die Antikörper-Produktion, so daB die IgM-Konzentration im Fermenter konstant bleibt. Erst der Zusatz von $500 \mu \mathrm{mol} / \mathrm{l}$ Serin in das Perfusionsmedium ab Tag 12 führt zum erneuten Anspringen der IgM-Produktion. Dadurch konnte die anfängliche spezifische Produktionsrate an Antikörper fast wieder erreicht werden, und die Antikörper-Konzentration im Fermenter erreichte einen Spitzenwert von $1,5 \mathrm{~g} / \mathrm{l}$.

Durch die kontinuierliche Zellernte sinkt dann die IgM-Konzentration wieder ab, die Produktion bleibt allerdings in Gang.

\section{Diskussion}

Die vorgestellten Ergebnisse demonstrieren einerseits, daß das Fermentationskonzept mit blasenfreier Begasung, Mikrofiltrationsmembran im Fermenter und Ultrafiltrationsmembran außerhalb des Fermenters eine funktionsfähige, aber auch flexible Einheit bildet, und 
andererseits, daß für eine gute Fermentationsführung ausreichende Kenntnisse der Bedürfnisse der jeweiligen Zellen vorhanden sein müssen.

Die Stabilität des Systems konnte bis jetzt auf bis zu 2 Monate Perfusionsbetrieb gesteigert werden (Daten hier nicht gezeigt, vgl. dazu [1]). Dabei ist allerdings unbedingt zu beachten, daß die Perfusionsrate keinesfalls wesentlich über den Richtwert von 1 Liter pro Meter Membran und Tag gesteigert wird. Höhere Perfusionsraten führen in der Regel sehr schnell (innerhalb einiger Tage) zu einer totalen Verblockung der Poren der hydrophilisierten Membran. Zum Erreichen höherer Zelldichten bei dieser maximalen Perfusionsrate kann einerseits die Membranlänge (eventuell segmentiert) vergrößert, andererseits das Perfusionsmedium nach den Erkenntnissen aus den Substratanalysen speziell supplementiert werden. Diese zweite Möglichkeit kann aus Gründen der Osmolarität und der durch Zusatz an Substraten erhöhten Produktion von Abfallstoffen wie Lactat, Alanin, Glutamat, $\mathrm{NH}_{3}$ usw., die entsorgt werden müssen, nicht in unbegrenztem Maße genutzt werden. In der Regel ist es deshalb notwendig, den nachwachsenden Teil der Zellmasse batchweise oder bevorzugt kontinuierlich aus dem Fermenter zu entfernen. Dies sollte so geschehen, $\mathrm{da}$ eine Limitierung (falls sie sich wie in diesem Fall negativ auswirkt) nicht auftreten kann.

Die Aufbringung der Membranen auf den Rührer hat den wesentlichen Vorteil, da $B$ ein Bewuchs durch Zellen bzw. eine Ablagerung von Proteinen fast ausgeschlossen ist. Vergleichende Untersuchungen mit einer starr eingebrachten, hydrophilisierten Membran ergaben starken Zellbewuchs und zusätzlich Bildung einer sichtbaren Deckschicht aus Protein (Albumin), die zu einem undefinierten Ultrafiltrationsverhalten der Mikrofiltrationsmembran führte.

Der Maßstab dieses Fermentationssystems wurde bis auf ein Arbeitsvolumen von 1001 gesteigert. Eine Fermentation derselben Zellinie im 23-l-Maßstab ergab zu den hier gezeigten Ergebnissen identische Resultate [4].

Eingegangen am 27. Dezember 1989 [K 1128]

\section{Literatur}

[1] Büntemeyer, H.; Bödeker, B. G. D.; Lehmann, J.: Membranestirrer-reactor for bubble free aeration and perfusion, in: Modern Approaches to Animal Cell Technology, Eds. R. E. Spier and J. B. Griffiths, Butterworth, London 1987, S. 411/419.

[2] Lehmann, J.; Vorlop, J.; Büntemeyer, H.: Bubble free reactors and their development for continuous culture with cell recycle, in: Animal Cell Biotechnology, Eds. R. E. Spier and J. B. Griffiths, Academic Press, London 1988, Vol. 3, S. 221/237.

[3] Büntemeyer, H.; Lehmann, J.: Vorrichtung und Verfahren zur blasenfreien Begasung von Flüssigkeiten, insbesondere von Kulturmedien zur Vermehrung von Gewebekulturen, PCT/EP 86.00 566 und $P 3535$ 183.7, 1986, 1987.

[4] Bödeker, B. G. D.; Hewlett, G.; Schlumberger, H. D.; Büntemeyer, $H$.; Lehmann, J.: Verfahren zur Kultivierung von Säugerzellen in einer Suspensionskultur, P 3541 826.9, 1986.

[5] Büntemeyer, H.: Dissertation, Univ. Hannover 1988.

\section{Aspekte bei der Realisierung kooperierender Expertensysteme zur ProzeBgenerierung}

\section{Hans-Dietrich Engelmann und Ralf Funder*}

\section{Herrn Professor Dr. Karl-Hans Simmrock zum 60. Geburtstag}

Die systematische Generierung von verfahrenstechnischen Prozessen gilt seit jeher als ausgesprochen kreatives Arbeitsgebiet. Wir versuchen im Sinne der „Strategischen Prozeßsynthese“ das, was man mehr oder weniger intuitiv bei der Entwicklung einer Prozeßstruktur ausführt, methodisch zu durchleuchten. Wegen der vielen, gleichzeitig zu berücksichtigenden Kriterien und der zahlreichen Informationen, die zu verarbeiten und teilweise auch erst während des Synthesevorganges zu berechnen sind, wurden am Lehrstuhl Technische Chemie A der Univ. Dortmund verschiedene wissensbasierte Systeme entwikkelt.

Das Beispiel der thermischen Crackung von Naphtha (und böheren Kohlenwasserstoff-Schnitten) zur Herstellung von Ethylen zeigt [1], $\mathrm{da} \beta$ die Generierung eines Verfahrensfließbildes eine umfangreiche Wissensbasis erfordert, insbesondere dann, wenn - wie im vorliegenden Beispiel - mehr als ein einziges Trennverfahren zur Lösung des Problems benötigt wird (Abb. 1).

Eine Analyse der strategischen Vorgehensweise bei der Synthese eines derartigen Prozesses zeigt, daB die Gesamtaufgabe zunächst in Teilprobleme zerlegt wird. Mit deren Lösung werden verschiedene

\footnotetext{
* Prof. Dr.-Ing. H.-D. Engelmann, Univ. Dortmund, FB Chemietechnik, Postfach 500500, 4600 Dortmund 50, und FH Ostfriesland, FR Prozeßautomatisierung, Constantiaplatz 4, 2970 Emden, Dipl.-Inform. $R$. Funder, Lehrstuhl für Technische Chemie A, Univ. Dortmund, Postfach 500500, 4600 Dortmund 50.
}

Experten beauftragt. Aus den vorgeschlagenen Lösungsmöglichkeiten werden erfolgversprechende Gesamtproblemlösungen generiert, die dann detaillierte Berechnungen, z. B. mit Simulatoren, ermöglichen.

Sollen Expertensysteme zur Lösung einer derartig komplexen Aufgabe eingesetzt werden, ist eine vergleichbare Vorgehensweise sinnvoll. Es ist nicht ein umfassendes System zu erstellen, das das gesamte Wissen enthält, wirkungsvoller ist der Einsatz von Teilsystemen für begrenzte Aufgabengebiete mit möglichst viel Domain-Wissen. Diese Überlegungen führten zu den kooperierenden verteilten Expertensystemen, d. h. einem Netzwerk lose gekoppelter Systeme, die zur Problemlösung miteinander kooperieren. Jedes System ist dabei für ein spezielles Teilproblem zuständig.

Zur Realisierung eines solchen Konzeptes stehen verschiedene Möglichkeiten zur Verfügung [2]. Viele theoretische Untersuchungen lassen sich derzeit für konkrete Anwendungen nicht umsetzen. Hier wird eine über das Blackboard-Konzept [3] hinausgehende Strategie verwendet.

Das Blackboard-Konzept selbst sieht ein Managersystem (Scheduler) vor, das verschiedene Wissensquellen (Knowledge Sources) aktiviert, wobei die gesamte Kommunikation über eine Wandtafel (Blackboard) abläuft. Dabei kann eine Wissensquelle als eine logisch zusammengehörige Regelmenge betrachtet werden, deren Auswirkung in einer gemeinsamen Datenstruktur, der Wandtafel, verzeichnet wird. Ihr Inhalt wird von dem Managersystem interpretiert. Bei Bedarf ruft das Managersystem eine weitere Wissensquelle auf. Diese verändert den Inhalt der Blackboard so, daß der neue Eintrag der Problemlösung näher kommt.

Die Weiterentwicklung dieses Konzeptes [4] verdeutlichen die folgenden Gegenüberstellungen:

1) In den Blackboard-Systemen findet keine direkte Kooperation zwischen den Wissensquellen statt. Es erfolgt eine gemeinsame Nutzung der auf der Wandtafel verzeichneten Informationen und Teillösungen, um auf ihr das Ergebnis zu erarbeiten. 